\title{
Functional Diagnosis in Upper and Lower Gastrointestinal Diseases: Relevance for Conservative, Interdisciplinary and Surgical Therapies
}

\author{
Martina Müller-Schilling ${ }^{\mathrm{a}} \quad$ Ines Gockel $^{\mathrm{b}}$ \\ a Department of Internal Medicine I, University Hospital Regensburg, Regensburg, Germany; \\ ${ }^{b}$ Department of Visceral, Transplant, Thoracic and Vascular Surgery, University Hospital of Leipzig, Leipzig, Germany
}

Recent years have seen dramatic improvements and developments in the clinical investigation of digestive functions.

The current special edition of Visceral Medicine provides a comprehensive overview of the full spectrum of functional gastrointestinal evaluation with emphasis on interdisciplinary management as well as conservative and surgical approaches. Only with the introduction of new diagnostic methods, which signify the high dynamic input of innovation in this area, new endoscopic and surgical treatment modalities have recently been enabled. These are portrayed here by means of evidence-based aspects, presented by experts in their respective field and written for our daily practice. This special edition considers highly differentiated perspectives on topics which are rarely discussed elsewhere at an elaborated level but are of general interest for patients with functional disorders. The contents of this focus share an educational and practical common thread with each highly-focused chapter, thus comprehensively addressing pertinent clinical challenges of this complex topic.

As a result, this special issue of Visceral Medicine covers pharyngeal and esophageal motility disorders, non-cardiac chest pain (NCCP), gastroesophageal reflux disease (GERD), gastric and digestive function, as well as anorectal disorders.

We have asked our experts to review the progress in the clinical measurement of gastrointestinal function, including high-resolution esophageal manometry, multiple intraluminal impedance, and advanced tests of anorectal function, as the fundament of new conservative, interdisciplinary, and surgical therapeutic strategies.

NCCP is recurrent angina pectoris-like pain without evidence of coronary heart disease in conventional diagnostic evaluation. Worldwide, the proportion of NCCP among patients with chest pain is reported between 20 and $40 \%$. The proportion of NCCP in emergency departments and chest pain units is even higher and ranges between 60 and $80 \%$. In contrast to the defined process guidelines for the management of patients with cardiac chest pain (CCP), a structured workup after exclusion of CCP is still missing. Frieling [1] suggests a diagnostic algorithm for the diagnosis of NCCP from a gastroenterologist's point of view. Gastroenterological expertise is required because in $50-60 \%$ of cases GERD, in 15$18 \%$ hypercontractile esophageal motility disorders, and in 32-35\% other esophageal alterations may be detected as the cause of chest pain.

GERD is one of the most common diseases worldwide with up to $20 \%$ suffering from weekly symptoms. Recently, an international group of experts (International Working Group for Disorders of Gastrointestinal Motility and Function) updated the Porto consensus and established recommendations on the indication and interpretation of esophageal function testing for the evaluation of GERD in clinical practice. This new consensus opinion is reviewed by Kandulski et al. [2] and Keller [3].

The article by Koop [4] focuses on recent advances in the medical therapy of GERD and advocates for a broader concept in the non-surgical management of GERD, which includes treatment strategies beyond proton pump inhibitors.

Surgery for benign esophageal disease - GERD and achalasia being the most frequent indications - is sophisticated and demanding at the same time. Thus, indications for surgery have to be highly elaborated with differentiated and structured algorithms, based on objective functional workup in the esophageal laboratory - always in combination with the patient's history and the respective symptom scores. Not only short- and long-term complications or adverse effects following functional esophageal surgery but also false indications and inadequate technical follow-up may lead to a deteriorating quality of life.

Functional outcome is of the utmost interest and has to be driven by the need for comprehensive but purposeful diagnostic methods. Selection from the diagnostic 'toolbox' before and after

\section{KARGER}

(c) 2018 S. Karger GmbH, Freiburg
Prof. Dr. Martina Müller-Schilling Klinik und Poliklinik für Innere Medizin I Universitätsklinikum Regensburg Franz-Josef-Strauß-Allee 11, 93042 Regensburg, Germany martina.mueller-schilling@ukr.de 
esophageal surgery becomes more difficult in the context of economic pressure, as e.g. the German DRG (= Diagnosis Related Groups) system does not adequately mirror the personal efforts, time resources, and materials used for the esophageal laboratory in the ambulatory setting. As not each patient can be provided with the complete 'toolbox' of functional investigations, proper selection is the key to successful therapy and long-term follow-up. The adequate assessments are discussed in the reviews by Keller [3] and Gockel et al. [5].

Chronic constipation is a very common medical problem, and - depending on the definition - up to $15 \%$ of the general German population report symptoms of constipation. Prevalence rates increase with age. The article by Andresen and Layer [6] covers the new Rome IV definition, current treatment standards, as well as future treatment options. Thus, a variety of primary and secondary disturbances of bowel function (motility, secretion, sensitivity) or of defecation may lead to constipation. The authors state that the establishment of clinical guidelines may not only improve treatment strategies but also 'the recognition of constipation as a relevant medical problem for many patients'.

Anorectal surgery covers a wide spectrum of surgery from operations for hemorrhoids to intersphincteric resection for low rectal cancer. Witte et al. [7] as well as Heinrich and Misselwitz [8] discuss the information needed from the functional laboratory before and after surgery. Furthermore, Heinrich and Misselwitz [8] place emphasis on the role of high-resolution anorectal manometry as a promising new tool in the diagnosis of functional anorectal disorders.

In summary, functional diagnosis in upper and lower gastrointestinal diseases is highly sophisticated and has recently gained increasing interest due to its enormous relevance for further therapeutic strategies. New diagnostic tools and an increasing understanding of gastrointestinal pathophysiology allow for more precise assessment and consecutive tailored treatments - aiming for an improved post-therapeutic quality of life. In this context, the development of innovative techniques leads to a closer and interdisciplinary link between gastroenterologists and surgeons with the common vision of excellence in quality, as exemplified by the 'Interdisciplinary Discussion' chaired by Fox [9].

The guest editors would like to express their gratitude and appreciation to the authors for their outstanding efforts, most valuable contributions, and excellent cooperation on this special topic on 'Functional Diagnosis in Upper and Lower Gastrointestinal Diseases: Relevance for Conservative, Interdisciplinary and Surgical Therapies'.

\section{Disclosure Statement}

A conflict of interest does not exist.

\section{References}

1 Frieling T: Non-cardiac chest pain. Visc Med 2018; 34:DOI: $10.1159 / 000486440$

2 Kandulski A, Moleda L, Müller-Schilling M: Diagnostic investigations of gastroesophageal reflux disease: who and when to refer and for what test? Visc Med 2018;34:DOI: 10.1159/000488184.

3 Keller J: What is the impact of high-resolution manometry in the functional diagnostic workup of gastroesophageal reflux disease? Visc Med 2018;34:DOI: $10.1159 / 000486883$.

4 Koop H: Medical therapy of gastroesophageal reflux disease beyond proton pump inhibitors: where are we heading? Visc Med 2018;34:DOI: 10.1159/000486692.
5 Gockel I, Rabe SM, Niebisch S: Before and after esophageal surgery: which information from the functional laboratory is needed? Visc Med 2018;34:DOI: $10.1159 / 000486556$

6 Andresen V, Layer P: Medical therapy of constipation: current standards and beyond. Visc Med 2018;34:DOI: $10.1159 / 000488695$.

7 Witte M, Schwandner F, Klar E: Before and after anorectal surgery: which information is needed from the functional laboratory? Visc Med 2018;34:DOI: $10.1159 / 000486693$
8 Heinrich H, Misselwitz B: High-resolution anorectal manometry - new insights in the diagnostic assessment of functional anorectal disorders. Visc Med 2018 34:DOI: $10.1159 / 000488611$.

9 Fox M, Heinrich H, Perretta S, Steinemann D: Functional diagnosis in upper and lower gastrointestinal diseases: relevance for conservative, interdisciplinary and surgical therapies (Interdisciplinary Discussion). Visc Med 2018;34:DOI: 10.1159/000489027. 